\title{
Benign Hepatic Portal Venous Gas in a Critically III Patient
}

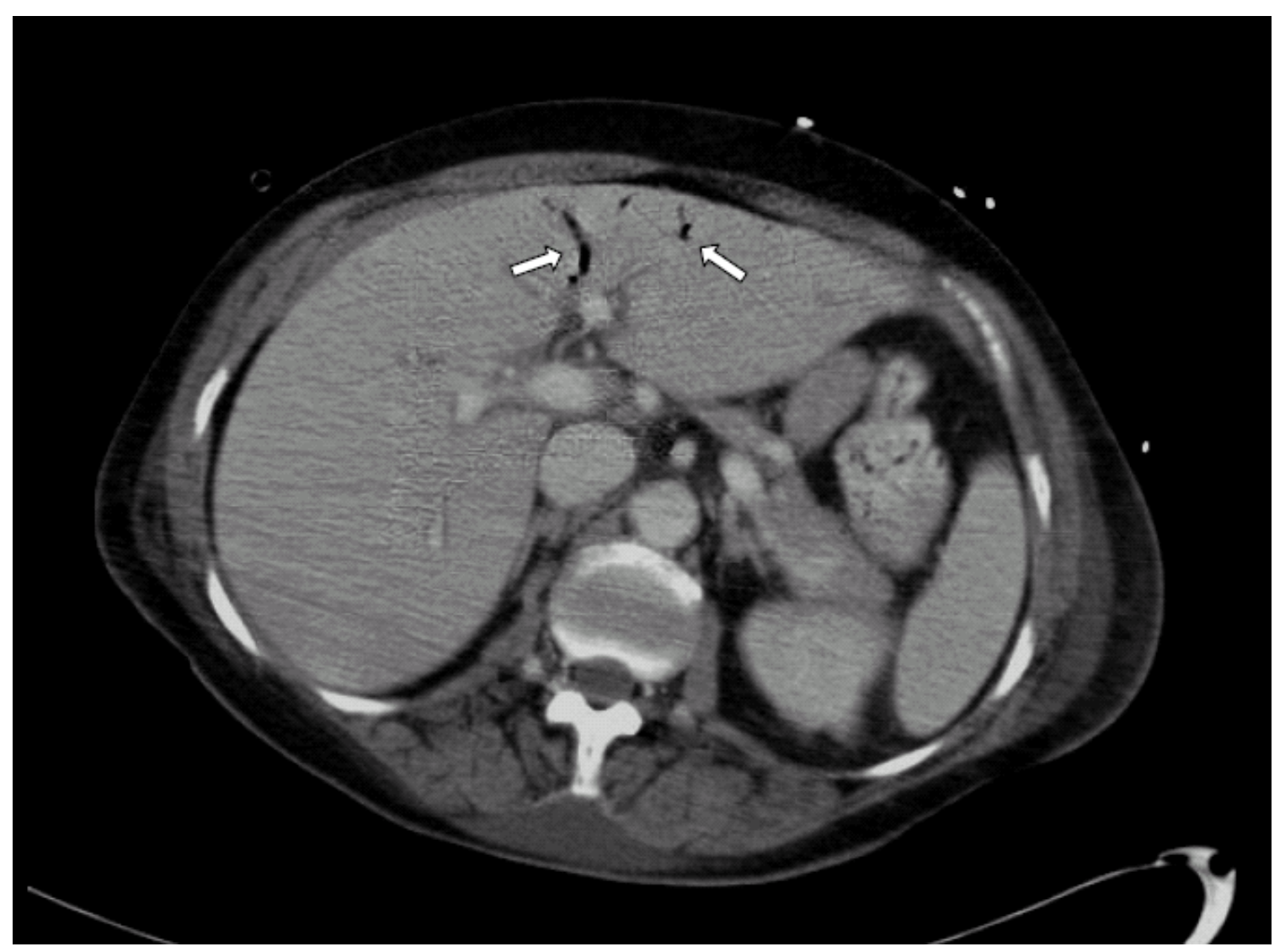

FIGURE. CT of the abdomen showing hepatic portal venous gas (see arrows).

\section{Emmanuel C. Gorospe}

Department of Medicine, Johns Hopkins Bayview Medical Center, Johns Hopkins University School of Medicine, Baltimore, MD

E-mail: egorosp1@jhmi.edu

Received March 31, 2008; Revised September 14, 2008; Accepted September 28, 2008; Published October 3, 2007

KEYWORDS: hepatic portal venous gas, bowel ischemia, computed tomography 
Hepatic portal venous gas (HPVG) formation is regarded as a sign of a serious intra-abdominal process due to its association with bowel necrosis[1], perforation[2], obstruction[3], and mesenteric infarction[4]. It is attributed to either disruption of intestinal mucosa, gas production in the hepatic portal system, or to iatrogenically increased intestinal lumen pressures such as in nasogastric intubation[4,5]. This abdominal CT scan is from a 62-year-old female who was admitted to the intensive care unit for hypercarbic respiratory failure from chronic obstructive pulmonary disease exacerbation. After 2 days of nasogastric tube placement, the patient's alkaline phosphatase increased from 84 to $210 \mathrm{U} / \mathrm{L}$. She has no history of recent endoscopic gastrointestinal examination. Her abdominal exam was unremarkable. The rest of the chemistry panel, including lactic acid, was within normal limits except for an elevated gamma-glutamyl transpeptidase of $419 \mathrm{U} / \mathrm{L}$. Blood cultures were negative. An abdominal CT scan revealed new HPVG formation as compared to an earlier scan on admission. There were no CT scan findings of free intraabdominal gas, pneumatosis intestinalis, bowel or mesenteric ischemia, lymphadenopathy, gall bladder disease, pancreatitis, bowel obstruction, or inflammation. Given these findings, no aggressive intervention was done. On hospital discharge, her liver enzymes normalized and follow-up abdominal ultrasound showed resolution of HPVG. Understanding that there are multiple conditions that can precipitate the development of HPVG, its finding should prompt a thorough diagnostic evaluation to immediately rule out a catastrophic intra-abdominal condition. The patient's overall clinical condition should be placed into perspective when deciding on the need for any invasive intervention.

\section{REFERENCES}

1. Gan, H.N., Tan, K.Y., Chong, C.K., and Tay, K.H. (2006) Finding hepatic portal venous gas in an adult patient: its significance. Singapore Med. J. 47, 814-816.

2. Duggal, A., Rankin, R.N., and Wall, W.J. (2007) Hepatic portal venous gas from perforated sigmoid diverticulitis. Can. J. Surg. 50, E19-20.

3. Hong, J.J., Gadaleta, D., Rossi, P., Esquivel, J., and Davis, J.M. (1997) Portal vein gas, a changing clinical entity. Report of 7 patients and review of the literature. Arch. Surg. 132, 1071-1075.

4. Monneuse, O., Pilleul, F., Barth, X., Gruner, L., Allaouchiche, B., Valette, P.J., and Tissot, E. (2007) Portal venous gas detected on computed tomography in emergency situations: surgery is still necessary. World J. Surg. 31, 1065 1071 .

5. Yamamuro, M. and Ponsky, J.L. (2000) Hepatic portal venous gas: report of a case. Surg. Today 30, 647-650.

This article should be cited as follows:

Gorospe, E.C. (2008) Benign hepatic portal venous gas in a critically ill patient. TheScientificWorldJOURNAL 8, 951-952. DOI 10.1100/tsw.2008.133. 


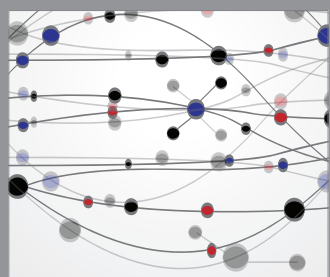

The Scientific World Journal
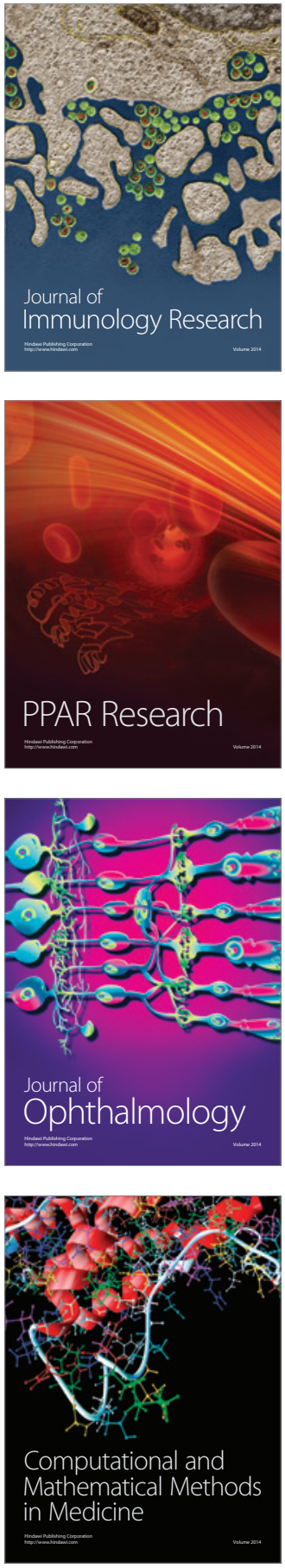

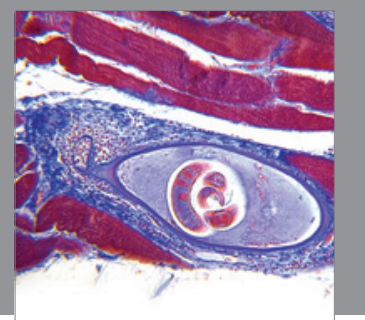

Gastroenterology

Research and Practice
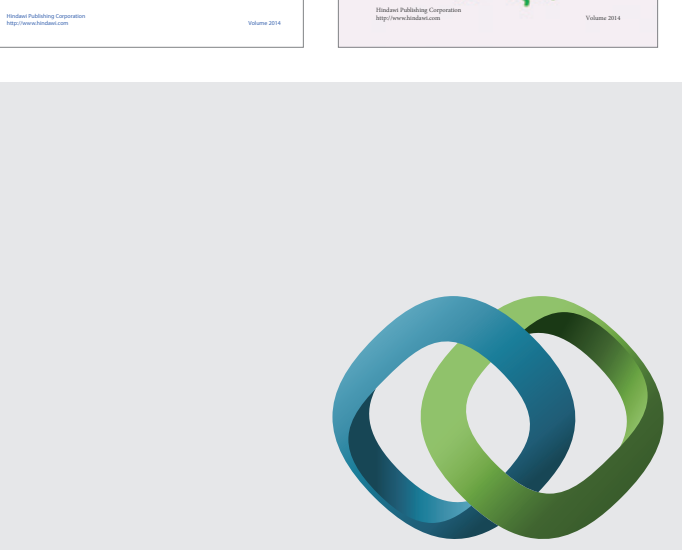

\section{Hindawi}

Submit your manuscripts at

http://www.hindawi.com
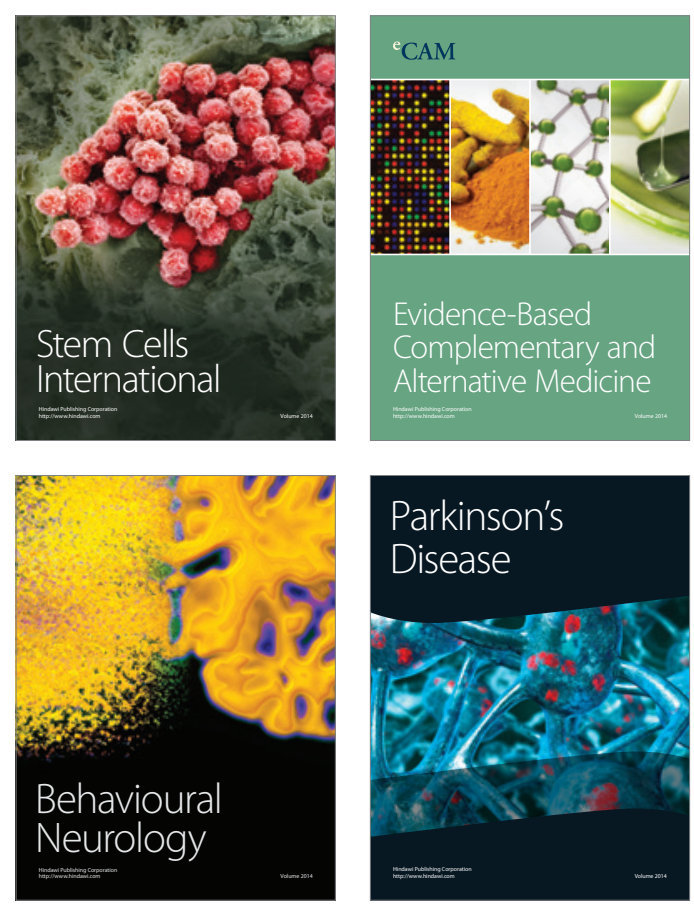

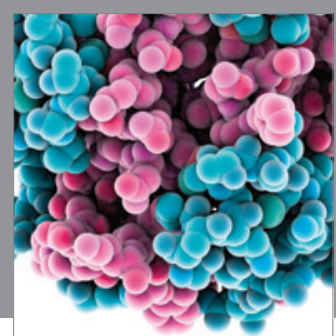

Journal of
Diabetes Research

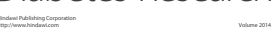

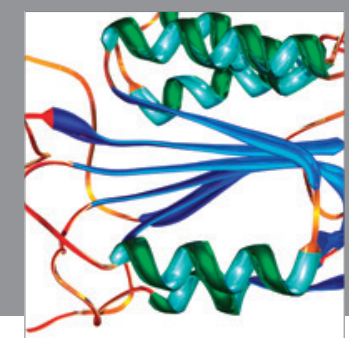

Disease Markers
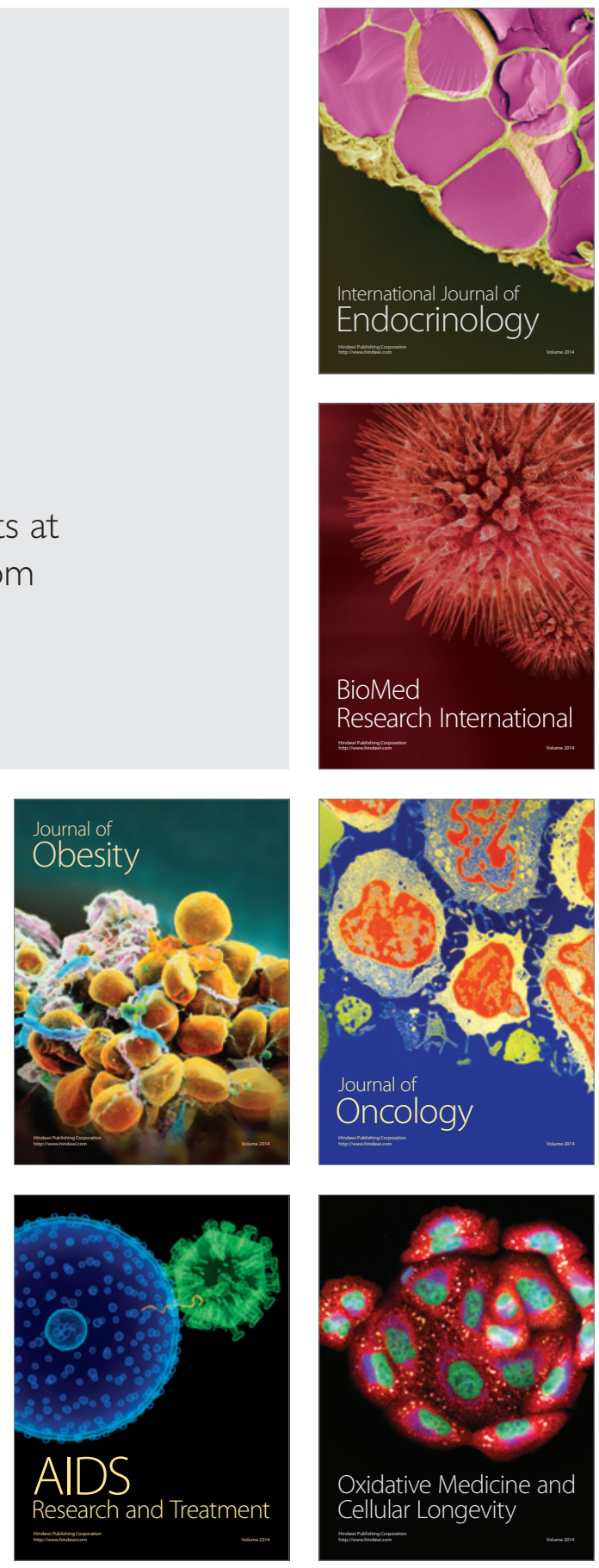